\title{
Chapter 2 \\ State of the Art and Perspectives of Ultrasound Imaging as a Human-Machine Interface
}

\author{
Claudio Castellini
}

\begin{abstract}
Medical ultrasound imaging is a diagnostic tool based upon ultrasound wave production, propagation and processing, in use since the 1950s in the hospitals all over the world. The technique is totally safe, relatively cheap, easy to use and provides live images of the interiors of the human body at both high spatial and temporal resolutions. In this chapter we examine its use as a novel human-machine interface. Recent research indicates that it actually represents an effective, realistic tool for intention gathering, at least for the hand amputees. Given the current state of the art, medical ultrasound imaging can be used to control an upper-limb prosthesis to a high degree of precision; moreover, the related calibration procedure can be made extremely short and simple, with the aim of building an ultrasound-based online control system. We propose and discuss its pros and cons as an interface for the disabled, we elaborate on its potentialities as a tool for intention gathering, and we show that it has great potential in the short- and mid-term.
\end{abstract}

Keywords Ultrasound imaging • Human-machine interfaces • Rehabilitation robotics

\subsection{Introduction}

Medical ultrasound imaging (from now on, US imaging) has nowadays become a standard diagnostic tool in hospitals. It is used to visualise essentially all human body structures and sports an enormous wealth of information, to the point that a trained specialist can diagnose a wide range of conditions just by looking at the live images it gathers. US imaging is also widely employed as a pre-operating tool along

C. Castellini, Ph.D. (ه)

Robotics and Mechatronics Center, German Aerospace Center, Oberpfaffenhofen, Germany

e-mail: claudio.castellini@dlr.de 
with other kinds of medical imaging such as, e.g., magnetic resonance and positron emission tomography; and during invasive procedures (interventional ultrasonography) to guide the insertion of operating tools into the body. (A comprehensive reference about US imaging, its foundations and physics, and even its therapeutic uses, can be found in [12].)

With respect to the above-mentioned imaging techniques however, it has at least three definite advantages: firstly, it does not entail direct radiation, nor any radioactive contrast means to be injected in the patient's body; this means that its level of safety is much higher - it is actually considered harmless [46]. Secondly, it enforces high imaging resolution, both spatial and temporal. Thirdly, the equipment to perform US imaging is, at least if compared to other techniques, extremely cheap and lightweight. US imaging machines are nowadays to be found in any hospital of any medium- to large-sized city; their buying and maintenance costs are low; and recently, hand-held US imaging machines have appeared on the market, as large and heavy as a smartphone, their cost hovering around a few thousand dollars.

From the point of view of rehabilitation robotics, what we are looking at is indeed a novel human-machine interface (HMI). The ideal HMI for this field must be cheap, lightweight, safe and rich in information; and so far, the only successful method is surface electromyography (sEMG from now on), with its applications to self-powered hand prosthetic control. Yet, sEMG is far from meeting all the abovementioned requirements: according to recent surveys [2,36,37], one quarter to one third of hand amputees reject prostheses controlled via sEMG, due to low reliability, weight, trouble with maintenance, low dexterity, and poor visual appearance. In fact, the sEMG signal can change due to several unpredictable factors - sweat, muscle fatigue, electrode displacement, etc. [7] - and is therefore far from being reliable.

We claim, and we will illustrate in the following, that medical ultrasound imaging has the potential to become an alternative, or additional, HMI for rehabilitation robotics. In this chapter we will first outline the historical development of medical ultrasound imaging as a diagnostic tool, which is rooted in the pioneeristic work of Karl Dussik in the 1940s. We will then sketch the basic functioning principles of the technique, and shorty report upon the current uses of pattern-matching technique in advanced ultrasound image processing, especially as far as prosthetics is concerned.

We will then move on to examine recent research leading to the idea of US imaging as a fully-fledged HMI for the disabled. In particular, the chapter revolves around the only two research efforts on this topic found so far in the community. Results therein indicate that (forearm) ultrasonography can detect the kinematic/dynamic configuration of the wrist and fingers with a high degree of finesse. The generality of the approaches presented lets us claim that similar results could be obtained in many other applications (e.g., lower-limb prosthetics). 


\subsection{Background}

\subsubsection{Historical Remark}

The first achievements in the direction of medical ultrasound imaging lie within the analytical theory of wave propagation as laid out in the works of Euler, D'Alembert and Lagrange in the eighteenth century. This theory contains the basis to interpret the reflection of a wave which has been intentionally emitted and propagates through a heterogeneous medium. Namely, it appeared from the propagation equations that each time a differential in the medium density and/or resistance to contraction was found, part of the energy of the wave would be reflected back - an echo would be produced.

Of equally high interest is the discovery of the piezoelectric effect in 1880 by Pierre and Jacques Curie. Piezoelectric materials, it was discovered, would react to the application of a voltage differential by contracting; if immersed in a suitable medium, they would propagate a mechanical wave through it. The piezoelectric effect worked the other way around, too: when pressure was applied to a piezoelectric material, a voltage differential at the surface of the material would arise. These materials could then be used as transducers: acting both as emitters of mechanical waves, and as receivers. These two strands of research came together, according to Cobbold [12], around 1916 with the work of Paul Langevin, Robert W. Wood and Alfred L. Loomis [21]. Research in this field was fueled, as it often happens, by a military application, namely the detection of submarines, which would later lead to the invention of the sonar; at the same time, the effects of ultrasound waves on biological tissue had been reported of by the above-mentioned authors. It was then clear that the emission/reflection of ultrasonic waves could in principle be used to inspect the internal structure of living beings.

The history of ultrasound as a medical device stems from these findings and officially begins, at least according to Kane et al. [31], in 1942, when Karl Dussik [17] attempted a full-breadth ultrasonic scan of the cranium of a brain tumour patient. In that paper, along with a theoretical treatment of the subject, fundamental considerations are made about the possibility of visualising several tissues at the same time, exploiting the above-mentioned principle of wave reflection at the interfaces. Figure 2.1 shows the apparatus Dussik built and used at that time.

In 1948, the first Congress of Ultrasound in Medicine was held in Erlangen, Germany; in 1955 the terms $A$-mode and B-mode ultrasound scanning (in turn, amplitude- and brightness-modes) were used for the first time, namely by John J. Wild at Cambridge. Lastly, in 1958 Ian Donald started using ultrasonic scanning as an aid to medical diagnoses in the case of an abdominal tumour; his pioneeristic result were published in Lancet [15]. Two years later, he developed the first two-dimensional ultrasonic scanner, and this is probably the point where modern ultrasound imaging is born. 


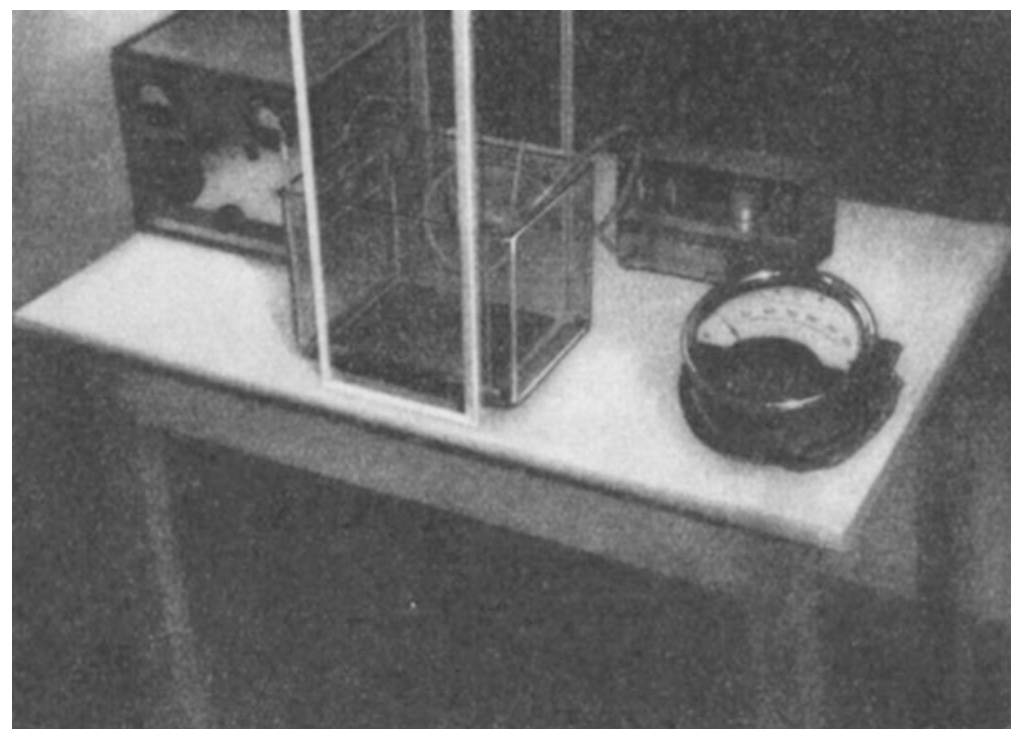

Fig. 2.1 Karl Dussik's apparatus to generate ultrasonic waves, built in 1942 (Reproduced from [17])

Musculoskeletal ultrasonography, that is, ultrasound scanning of bones, muscles and tendons, starts in 1958 again thanks to Dussik [18] who measured the acoustic attenuation of articular and periarticular tissues. To obtain a US image of a musculoskeletal structure though, one must wait until McDonald and Leopold's 1972 paper [34]; in which it is stated that two musculoskeletal conditions (namely, Baker's cysts and peripheral oedema) produce two different ultrasound patterns, which can be distinguished both from each other and from a healthy condition.

This is likely to be one of the first general diagnostic statements of a musculoskeletal condition based upon ultrasound imaging.

Although the basic principle has not changed, the performances of today's ultrasound machines are excellent under all points of view. Thanks to the blazing advancements in both piezoelectric technology, microelectronics and computer processing power, modern ultrasound machines can obtain full-resolution B-mode ultrasonic scans, that is, grey-valued images, of essentially any body structure. Current machines [28] reach temporal resolutions of up to 100 frames per second, spatial resolutions of $0.3-1 \mathrm{~mm}$ in the direction parallel to the transducer (the device leaning against the subject's skin), can visualise up to $15 \times 15 \mathrm{~cm}$ of tissue, and can be made so small that they are not bigger than a smartphone; there are examples of commercial hand-held ultrasound machines weighing $390 \mathrm{~g}$ with a $3.5^{\prime \prime}$ display. 

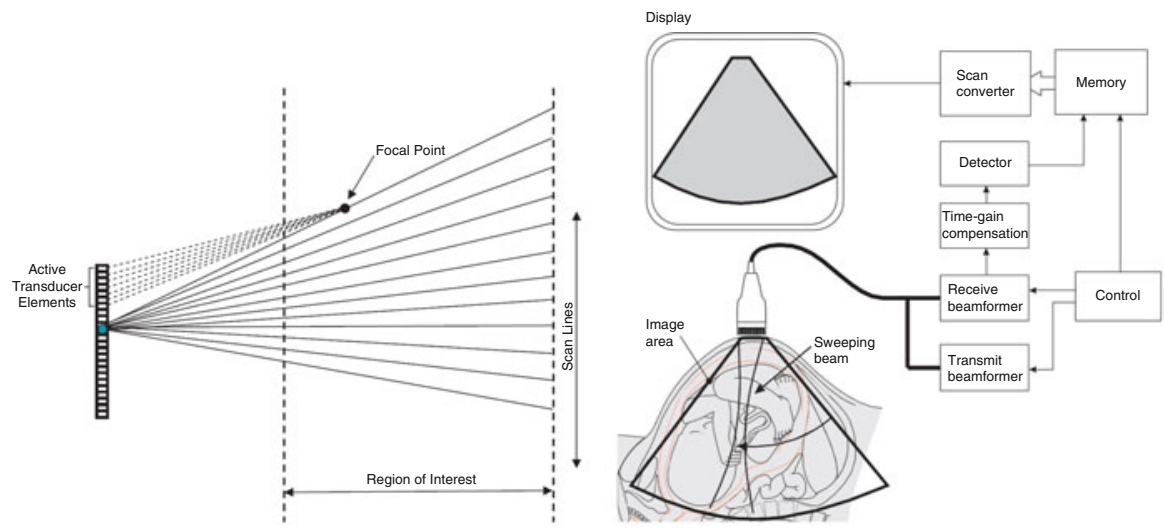

Fig. 2.2 (Left) A graphical representation of how ultrasound imaging works (Reproduced from [1]); (right) a scheme of the typical linear B-mode ultrasonographic device (Reproduced from [28])

\subsubsection{Technological Principles of Ultrasound Imaging}

A full mathematical treatment of (medical) ultrasound imaging can be found, e.g., in $[12,28]$; what follows is an informal description of the physics and technology underlying medical imaging. For a more technical description of how a medical ultrasonography device works, see, e.g., [1].

With the term ultrasound it is commonly meant sound waves of frequency over $20 \mathrm{kHz}$. The propagation speed $v$ of a sound (mechanical) wave in a physical medium depends on the mechanical characteristics of the medium itself according to the relation $v=\sqrt{\frac{B}{\rho}}$, where $B$ is the medium's adiabatic bulk modulus and $\rho$ is the medium's density. (The adiabatic bulk modulus measures the resistance of a substance to uniform compression.) Therefore, wherever a gradient in the medium's density or stiffness is found, $v$ will change and so will the energy associated to the wave. The differential in energy can in general result in wave absorption, transmission and reflection; in a real situation, all three phenomena will occur to different degrees, and the phenomenon will be particularly evident each time the wave hits the boundary between two different mediums.

As a result of that, any device able to measure the reflection of a definite wave after it has travelled back and forth in a region of interest will be able to determine what boundaries the wave itself has been travelling through before being attenuated beyond recognition. In the case of ultrasound waves, this principle lies beneath the ability of some animal species such as, e.g., bats to navigate flight and to locate food sources.

In the case of ultrasound imaging (see Fig. 2.2, left panel), an array of piezoelectric transducers is used to focus a multiplexed, synchronised set of ultrasound waves 
(beam) over a line lying at few centimeters' distance from the array. Each element of the array can, in turn, be used to convert the echo(es) of the emitted wave into a voltage. By accurately timing the echoes, one can determine the nature of the medium through which the wave has propagated; in particular, the echoes form the profile of the boundaries encountered along a straight line stretching away from the transducers. This information can be plotted, forming the so-called A-mode ultrasonography.

By employing a multiplexing device (beamformer), all transducers in the array can be synchronised to gather closely spaced profiles. The net result is a twodimensional representation of the section of the medium lying ahead of the array, or a so-called B-mode ultrasonography. (The array is often called the probe of the ultrasound machine, or, with a slight abuse of language, the transducer.) The intensity in the profiles, and therefore in their 2D juxtaposition (image) denotes rapid spatial variations in $B$ and/or $\rho$, therefore indicating abrupt changes of medium - interfaces. Figure 2.2 (right panel) shows a scheme of the typical B-mode ultrasonographic device: the ultrasound beam generated by the transmit beamformer sweeps the tissues of interest, generating reflections at each point in space where an interface between two tissues is found. Corresponding reflections are captured by the transducers and converted to a grey-valued image by a receive beamformer coupled with a time-delay compensation device. As a result, "ridges" in the image denote tissue interfaces.

The frequency selected for the ultrasound waves must be tuned, within a reasonable range, according to the tissue under examination and the required focus depth and depth of field; typically, wave frequencies are between 2 and $18 \mathrm{MHz}$.

\subsubsection{Ultrasound Imaging in Rehabilitation Robotics}

In rehabilitation robotics, a complex robotic artifact must be somehow interfaced with a disabled human subject. Depending on the task the subject requires and on which functionalities the robotic artifact provides, the rehabilitation engineer must figure out how to let the subject control the artifact to the best possible extent - the main issues here are those of reliability, dexterity and practical usability. An HMI lies therefore at the core of such a man-machine integration, and must be targeted on the patient's needs and abilities.

In particular, one must figure out, first of all, what kind of signals best suit the target application. The signals must be recognisable into stable and repeatable patterns, and must be produced by the subject with a reasonable effort, both physical and cognitive. Such patterns must then be converted into (feed-forward) control signals, i.e., motion or force commands for the robotic artifact. One early example of such a successful HMI is sEMG, initially developed as a diagnostic tool for assessing peripheral neural disorders and muscular conditions; from the 1960s on then, it was applied to control single-degree-of-freedom hand prosthesis, and its use 
has then progressed towards lower- and upper-limb self-powered prostheses [37]. More or less at the same time, advanced pattern recognition techniques started being used to convert the sEMG signal patterns into control signals, whenever the dexterity of the prosthesis called for a finer detection of the subject's intentions [14, 19,35].

The wealth of information delivered by medical ultrasound imaging, therefore, calls for the exploration of its use as a novel HMI in this field. The idea of applying machine learning techniques to ultrasound images in general is not new. Recent examples include, e.g., the recognition of skin cancer [30], tumor segmentation [50] and anatomical landmarks detection in the foetus [39]. But, as far rehabilitation robotics is concerned, the literature is scarce. To the best of our knowledge, at the time of writing the only application is that of $3 \mathrm{D}$ ultrasound imaging to the visualisation of residual lower limbs and to assess the ergonomy of lower-limb prostheses $[16,38]$.

\subsection{Sonomyography}

The first real use of US imaging as an HMI appears in 2006 and is due to Yong-Ping Zheng et al. [51]. The associated technique is called sonomyography. The authors focus on the large extensor muscle of the forearm, M. Extensor Carpi Radialis, and perform a wrist flexion/extension experiment on six intact subjects and three trans-radial amputees. In the study, two rectangular blocks are used to track the upper and lower boundaries of the muscle; tracking is enforced using a custom tracking algorithm based upon 2D cross-correlation between subsequent frames. The estimated muscle thickness is the distance between the centres of the two blocks. See Fig. 2.3.

The wrist flexion angle is determined using a motion tracking system and four markers placed on the subject's wrist and dorsum of the hand; in the case of the amputees, the subjects were asked to imagine flexing and extending the imaginary wrist while listening to an auditory cue (metronome). The correlation coefficient
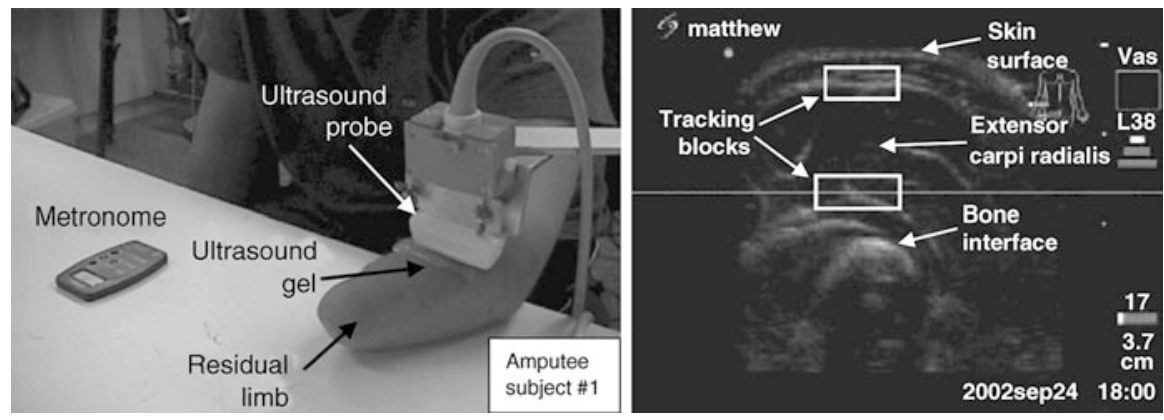

Fig. 2.3 Determining the wrist flexion angle via ultrasound imaging. (Left) The setup; (right) a typical US image (Reproduced from [51]) 
between the estimated muscle width and the wrist flexion angle is evaluated, showing high correlation in the cases of the intact subjects (linear regression average $R^{2}$ coefficient 0.875 ). A qualitative analysis of the results for the amputees is promising.

Extensive work on the same topic follows in a series of studies. In [26] high correlation is shown among wrist angle, sonomyography and the root-mean-square of the sEMG signal, which is well-known to be quasi-linearly related to the force exerted by a muscle [14]. The proposed approach is therefore validated by comparison with a standard technique and with the ground truth. In [42] a new algorithm is presented which further improves the previous results; lastly, in [23] a successful discrete tracking task controlled by sonomyography is presented. The subject pool is this time sensibly larger (16 intact subjects). As a followup, the feasibility of using sonomoygraphy to control a hand prosthesis has been demonstrated [10,11,42], but still limited to wrist flexion and extension. Attempts have been made to improve the estimation of wrist angle from sonomyography using support vector machine and artificial neural network models [24,47].

More recently, sonomyography has been successfully extended to elbow flexion/extension, wrist rotation, knee flexion/extension and ankle rotation [52]. Lastly, and this is probably the most interesting result so far [24], the approach is extended to A-mode ultrasonography: a wearable, single ultrasound transducer is used to determine the wrist flexion angle via a support vector machine $[3,13]$. A-mode ultrasonography is enforced by means of commercially available single ultrasound transducers applied on the subject's skin, much like sEMG electrodes. This opens up the possibility of miniaturising the sonomyography hardware and make it wearable. The issue of signal generation and conditioning is still open, though.

In the above-mentioned corpus of research, the authors have never considered more than one feature at the same time. This restricted focus is probably motivated by the diversity and complexity of the changes in US images as joint positions change: the single identified feature is related to a precise anatomical change, a relation which would be quite hard to assess in the general case. It is likely that a more general treatment in that case would require a detailed model of the kinematics of the human forearm, plus a detailed model of the changes in the projected US image as the hand joints move - a task which seems overtly complex. The only attempt so far at modelling finger positions appears in [43,44], where significant differences among optical flow computations for finger flexion movements are reported and classified.

\subsection{Ultrasound Imaging as a Realistic HMI}

The only other attempt at establishing US imaging as an HMI, as far as we know, is that carried on in our own group. In particular, with respect to sonomyography, this approach does not rely on anatomical features of the forearm (as visualised in 

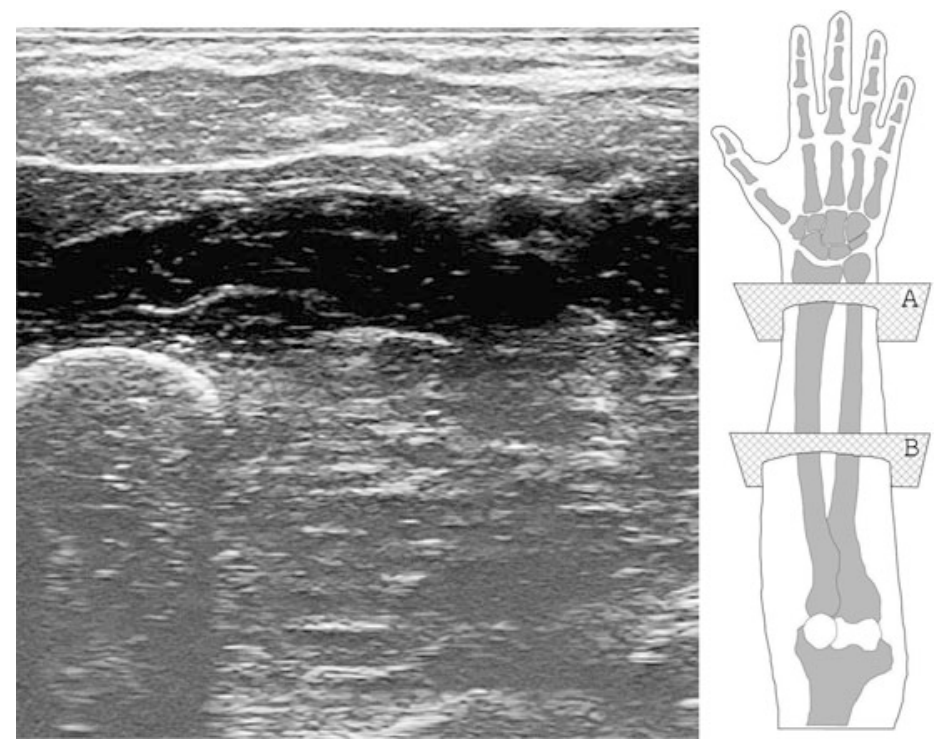

Fig. 2.4 (Left) A typical ultrasound image obtained during the qualitative analysis carried on in [5]. The ulna is clearly visible in the bottom-left corner, while the flexor muscles and tendons are seen in the upper part. (Right) A graphical representation of the human forearm and hand (right forearm; dorsal side up). The transducer is placed onto the ventral side; planes $A$ and $B$ correspond to the sections of the forearm targeted by the analysis

the ultrasonic images), but rather considers the images as a global representation of the activity going on in the musculoskeletal structure, as a human subject performs movement or force patterns with the hand. (This approach cannot therefore be consistently called "sonomyography", as the targeted structures are not restricted to muscles, but rather all organs are considered, i.e., veins and arteries, bones, nerves, connective and fat tissue, etc.)

\subsubsection{Ultrasound Images of the Forearm}

In $[5,49]$ the first qualitative/quantitative analysis of the ultrasonographic images of the human forearm, with respect to the finger movements, was carried out. Extensive qualitative trials were performed, i.e., live ultrasound images of sections of the human forearm were considered, while the subject would turn the wrist and/or flex the fingers, both singularly and jointly. An example is visible in Fig. 2.4.

One first result was that clearly, subsequent images related to each other in nontrivial ways. Human motion is in general enacted by contracting the muscles. When a muscle contracts, its length reduces but its mass must obviously remain constant; as a consequence, the muscle swells, mainly in the region where most muscle fibers 
are concentrated. This region, colloquially referred to as the muscle belly, expands in the directions orthogonal to the muscle axis; at the same time, the other organs in the forearm must accordingly shift around - this includes the bones, nerves, tendons and connective/fat tissue.

In the experiment, the ultrasound probe was held orthogonal to the forearm main axis, visually resulting in a complex set of motions: muscle sections (elliptical structures in the images) would shrink and expand; sometimes the muscle-muscle and muscle-tendon boundaries would disappear and reappear; and meanwhile, all other structures around would move in essentially unpredictable ways. It was clear that the deformations seen in the images could not easily be described in terms of simple primitives, such as, e.g., rotations, translations and contractions/expansions. Only very few simple features could have been explicitly targeted using anatomical knowledge, as it had been done in sonomyography; but as well, anatomy varies across subjects, which would have hampered the general applicability of the approach.

At the same time, it was apparent that whatever force the subject exerted (the three degrees of freedom of the wrist, the motion of single fingers down to the distal joints, etc.) was related point-to-point to the images. Muscular forces relate to torques at the joints, which in turn relate to measurable forces at the end effectors, for example at the fingertips. In the visual inspection there was no hysteresis effect, and each single dynamic configuration of the musculoskeletal structures under the skin would clearly correspond to a different image. This was the case both when the fingers would freely move and when they would apply force against a rigid surface or an object to be grasped. (Actually, that hinted at the fact that free movement actually represents the application of small forces, that is, those needed to counter the intrinsic impedance of the musculoskeletal structures.)

One direct consequence of this is that wrist and finger positions and forces could clearly be directly related point by point to each single image. This hinted at the possibility of using spatial features of the ultrasound images to predict the hand kinematic configuration (rather than, e.g., the optical flow or other features involving the time derivative of the images). This would have the advantage of being independent of the speed with which forces were applied.

Another very interesting characteristic of the forearm US images was that changes related to the hand/forearm kinematic configuration were almost totally local; e.g., it was tested that when the subject flexed the little finger, deformations would happen almost only in a certain region of the images; the region is loosely determined by the underlying anatomy. If, e.g., the US transducer was placed on the ventral side of the wrist, orthogonal to the axis of the forearm, then the action would be limited almost exclusively to the tendon leading out of the large flexor muscle (M. Flexor Digitorum Superficialis) controlling the little finger. The tendon pulls backwards and forwards parallel to the forearm axis, and appears as an elliptic structure growing and shrinking in a corner of the images. This further suggested the use of local spatial features of the images. 

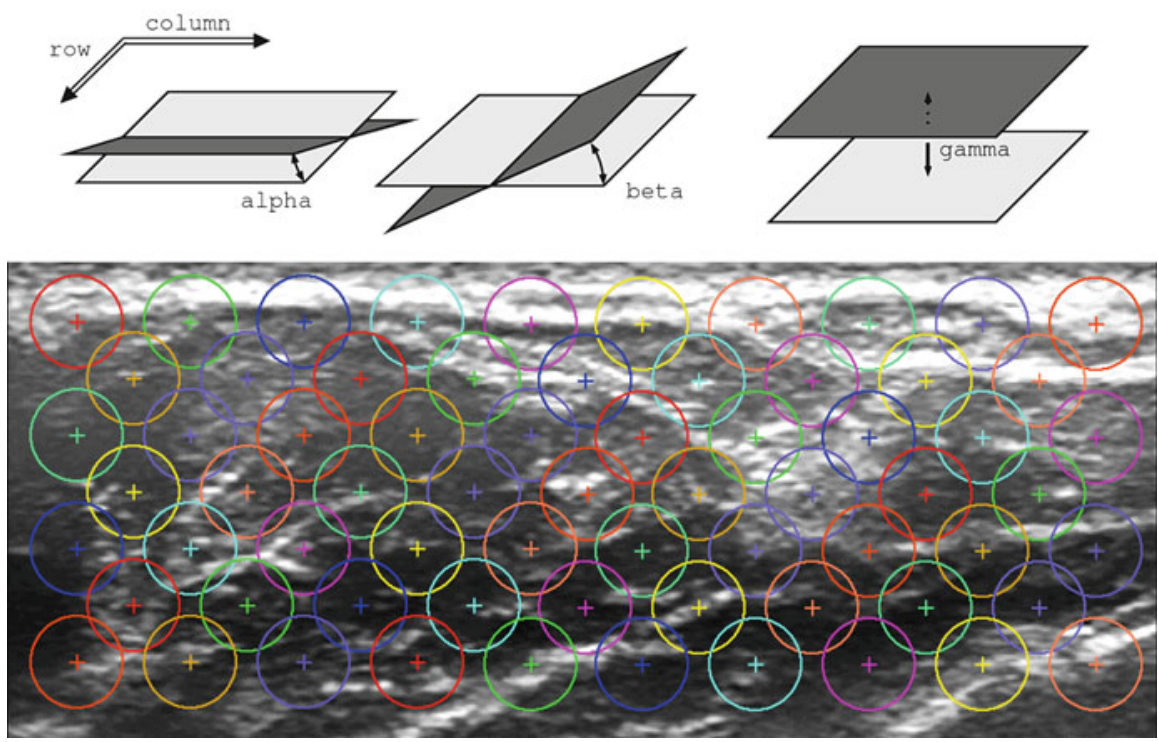

Fig. 2.5 (Above) A graphical representation of the meaning of the spatial features $\alpha_{i}, \beta_{i}, \gamma_{i}$; (below) the grid of interest points, as laid out on a typical US image (Reproduced from [49])

\subsubsection{Relating US Images and Finger Angles/Forces}

In the above-mentioned paper and in the subsequent [8], it was established that linear local spatial first-order approximations of the grey levels are linearly related to the angles at the metacarpophalangeal joints of the hand, leading to an effective prediction of the finger positions. More in detail, a uniform grid of $N$ interest points, $p_{i}=\left(x_{i}, y_{i}\right), i=1, \ldots, N$, was considered; a circular region of interest (ROI) of radius $r>0$, centered around each interest point, would then be determined:

$$
\mathrm{ROI}_{i}=\left\{(x, y):\left(x-x_{i}\right)^{2}+\left(y-y_{i}\right)^{2} \leq r^{2}\right\}
$$

Lastly, for each ROI, a local spatial first-order approximation of the grey values of its pixels $G(x, y)$ was evaluated:

$$
G(x, y) \approx \alpha_{i}\left(x_{i}-x\right)+\beta_{i}\left(y_{i}-y\right)+\gamma_{i}
$$

for all $(x, y) \in \mathrm{ROI}_{i}$. Intuitively, $\alpha_{i}$ denotes the mean image gradient along the $x$ direction (rows of the image), $\beta_{i}$ is the same value along the $y$ (columns) direction, and $\gamma_{i}$ is an offset. Figure 2.5 shows the grid of points and graphically illustrates the meaning of these features.

The above remark about the locality of the image changes is reflected in the changes in the feature values: Fig. 2.6 shows a correlation diagram, obtained by 


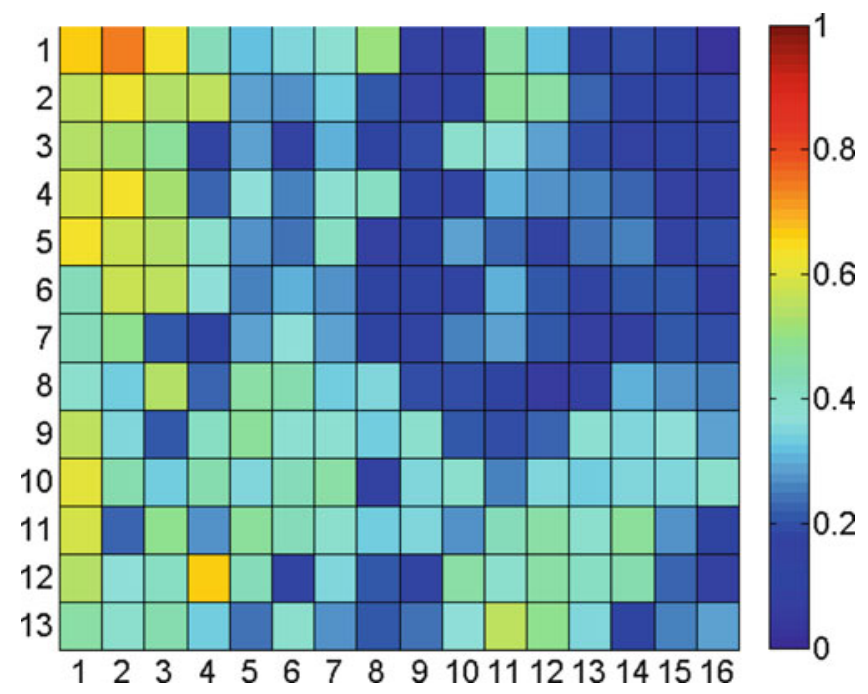

Fig. 2.6 Correlation diagram of the movement of the little finger. Each entry in the matrix denotes, for each region of interest $i$, the Pearson correlation coefficient between $\frac{\alpha_{i}+\beta_{i}+\gamma_{i}}{3}$ and the dataglove sensor values obtained while moving the little finger

considering the Pearson correlation coefficient between the average of $\alpha_{i}, \beta_{i}, \gamma_{i}$ and values of the sensor of the little finger of a dataglove (experiment reported in [8]). As is apparent from the figure, the movement of the little finger is strongly correlated to the feature values in the upper-left corner of the images.

Consider now the metacarpophalangeal angles of a finger, $\theta^{M C P}$; if the abovementioned features are considered, then

$$
\theta^{M C P}=\mathbf{w}^{T} \mathbf{v}
$$

where $\mathbf{v}$ is the vector of spatial features, and $\mathbf{w}^{T}$ is a vector of coefficients that can easily be estimated using, e.g., least-squares regression [4]. In general, given $n$ (sample,target) pairs $\left\{\mathbf{x}_{i}, y_{i}\right\}_{i=1}^{n}$, the optimal $\mathbf{w}$ is

$$
\mathbf{w}=\left(X^{T} X\right)^{-1} X^{T} \mathbf{y}
$$

where the matrix and vector $X, \mathbf{y}$ are formed by juxtaposing all samples and target values. This is the exact solution to the problem, and it involves inverting the $d \times d$ matrix $X^{T} X$, where $d$ is the dimension of $\mathbf{v}$. The task is therefore dominated by $d$ rather than by the number of samples used to estimate the relationship, $n$, which is usually much larger than $d$.

The claim of linearity was validated in $[5,8]$ by collecting data from 10 intact subjects and having them freely move the fingers while recording the US images of the wrist, and the finger angles using a dataglove. A rough 3D hand model on a 


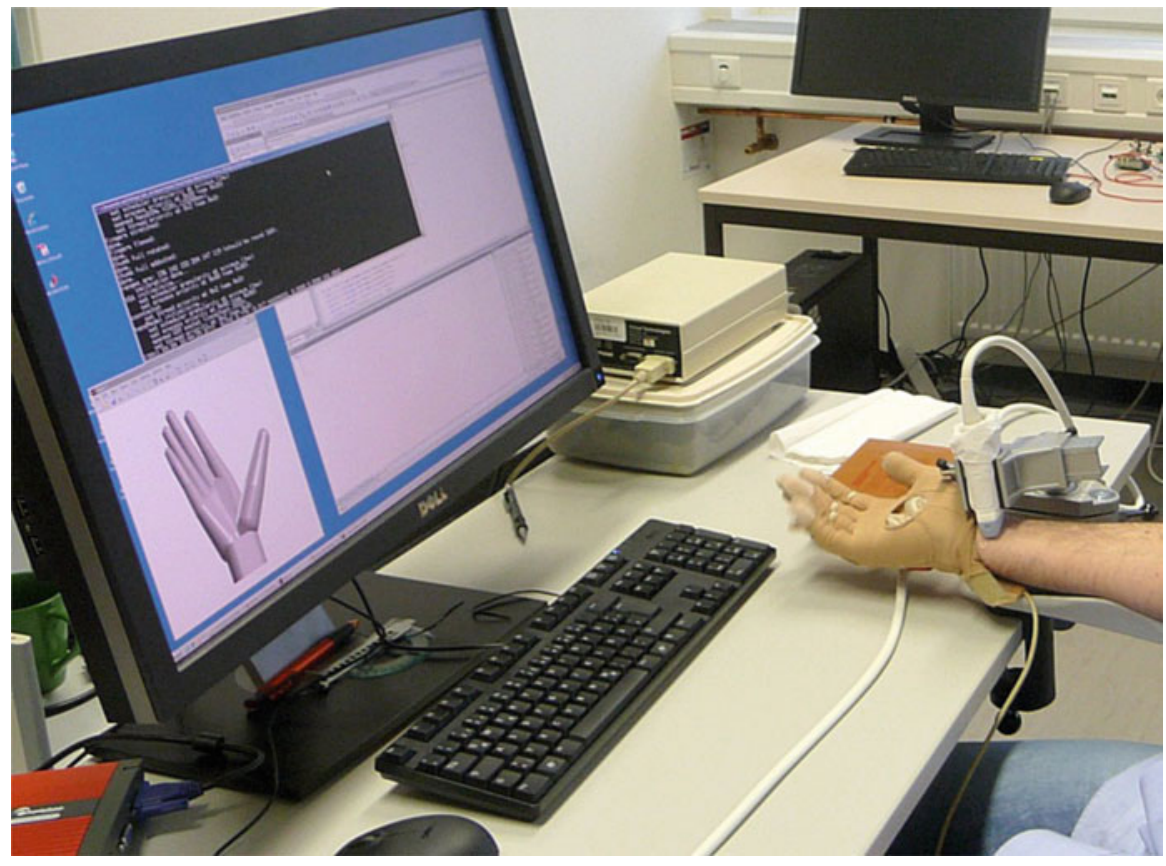

Fig. 2.7 The experimental setup used in $[5,8]$ to record US images and metacarpophalangeal angles. A 3D hand model would teach the subject the motion to imitate; the ultrasound probe was held against the subject's wrist using a vise; and a dataglove was used to collect the MCP joint angles

computer screen would show the subject the visual stimulus, inducing by imitation a certain motion of a finger. (Figure 2.7 shows the setup used therein.)

The regression error, evaluated as the root-mean-square error normalised over the dataglove sensor range, was found to be as little as 1-2\%. The accuracy analysis was extended in [49] (see Fig. 2.8) to the case in which fewer ROIs were selected, or in the case the radius of the ROIs, $r$, changed. It was discovered that the error was not very sensitive across a large range of radius values of the ROIs, but rather sensitive to the spacing of the grid.

In $[6,45]$, the analysis was extended to finger forces, thanks to the use of a force sensor. The same features were extracted from the ultrasound images, and the same technique was applied to obtain a regression map between forces $\mathbf{f}$ and spatial features, obtaining for each finger force $\mathbf{f}^{\text {FINGER }}$ a relationship

$$
\mathbf{f}^{F I N G E R}=\mathbf{w}^{T} \mathbf{v} .
$$

where $\mathbf{w}$ was found by a regularised variant of the least-squares regression method called ridge regression. With respect to Eq. 2.1, in this case

$$
\mathbf{w}=\left(X^{T} X+\lambda I_{d}\right)^{-1} X^{T} \mathbf{y}
$$



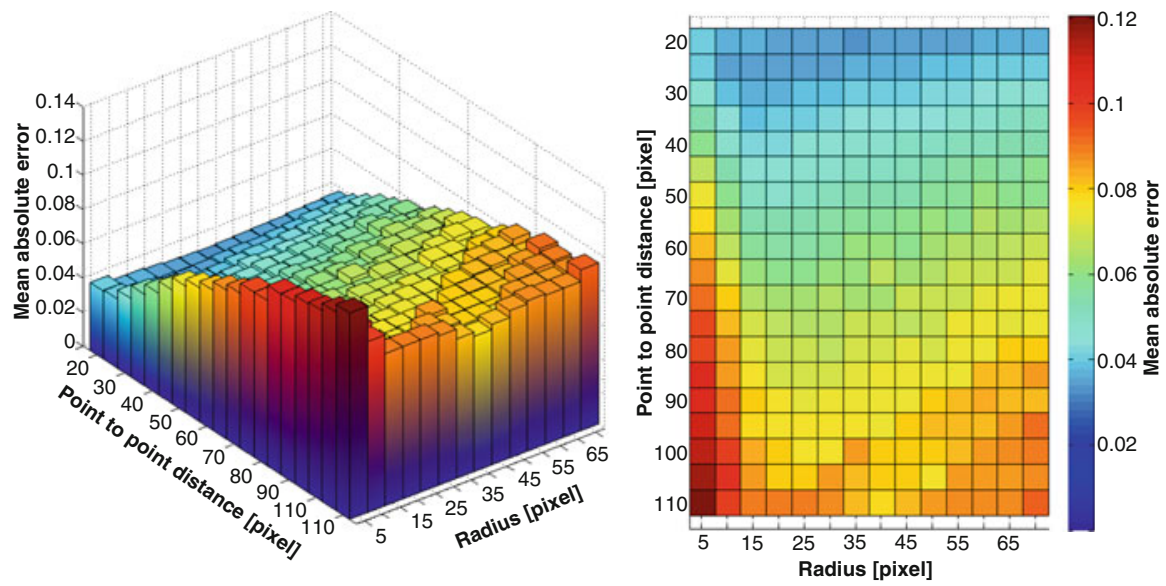

Fig. 2.8 Error analysis as the number of features considered changes, namely as the inter-ROI distance and ROI radius is varied

where $I_{d}$ is the identical matrix of dimension $d \times d$ and $\lambda>0$ is the regularisation coefficient, which was consistently set at the standard value of 1 . (It was verified that altering $\lambda$ would not change the results.)

In an experiment involving 10 intact subjects, it was assessed that the approximation obtained using Eq. 2.2 could predict all finger forces with a maximal error of about $2 \%$ of the sensor signal range.

\subsubsection{A Realistic Implementation}

The feasibility of the approach was further verified in [6,45], by setting up a realistic experimental scenario for this novel HMI. A minimal set of requirements was assessed for this technique to be applied to hand amputees; namely, (1) that the training (calibration) phase be short; (2) that it entail simple imitation tasks; (3) that it need no sensors; and lastly, (4) that the system be able to acquire new knowledge when required. These requirements are in general motivated by the bad condition of an amputee's stump, which quickly elicits fatigue and stress; moreover, amputees obviously the lack sensory feedback from the missing limb, which makes it hard (if not impossible) for the amputee to apply graded forces. Lastly, there is in principle no way of gathering ground truth, since no force sensors and/or datagloves can be used. The fourth requirement is motivated by the necessity of retraining previous patterns in case the signal changes due to, e.g., movement of the ultrasound transducer, or in order to improve the current prediction in case the subject is unsatisfied with it. Notice, anyway, that the vast majority of amputees have phantom feelings that do not correspond to the intended force/movement patterns; 


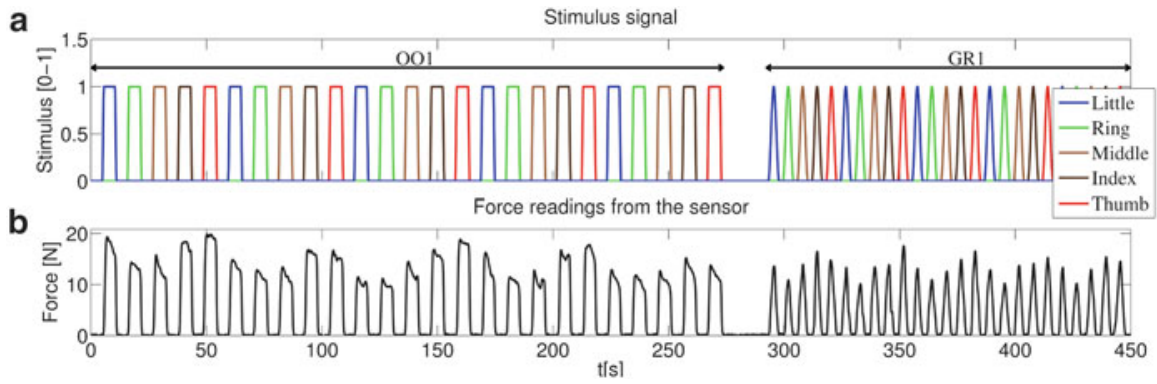

Fig. 2.9 (a) Part of the stimulus administered to the subjects in the experiment of $[6,45]$. In an "on-off" phase (OO1), only rest and maximum force were induced for each finger; in the graded phase (GR1) the subjects had to exert force following a squared sinusoidal pattern. (b) Typical forces, as actually measured by a force sensor during the experiment

therefore a further effort is required to ignore the feeling and this further motivates the requirement for a simple calibration task.

The realistic scenario set up in the above-mentioned papers consisted of a psychophysical task which only entailed resting and pressing with maximum force on a table, following a simple visual stimulus, the so-called "on-off" training. (A force sensor was actually used just to compare the results with the actual ground truth.) The regression machines so obtained were then tested on data obtained while following a fully-fledged sinusoidal pattern of finger forces, and observing whether training on the visual stimulus only would entail a remarkable loss in performance.

The stimuli were organised in two identical sessions, and each session was likewise divided in two parts, according to the kind of stimulus administered: an on-off phase (OO) and a graded phase (GR). The complete structure of the stimulus for one of the sessions is displayed in Fig. 2.9.

The results showed that this was not the case: on-off training could be employed to predict graded forces, and training on a visual stimulus produced errors (around $10 \%$ ) of the same order of magnitude of those obtained using the sensors force values. See Fig. 2.10.

\subsubsection{Incremental Learning}

Lastly, the fourth requirement of Sect. 2.4.3 was enforced by exploiting the linearity of the relationships found. Ridge regression was here implemented in its incremental variant (incremental or recursive ridge regression in the signal processing community, see [41]), in which the regression vector(s) $\mathbf{w}$ can be updated as soon as a new (sample,target) pair is available, and no complex computational operation is required. As a result, the system was demonstrated to work seamlessly at $30 \mathrm{~Hz}$ and could acquire new knowledge whenever required, de facto blurring the 
a ground truth: force
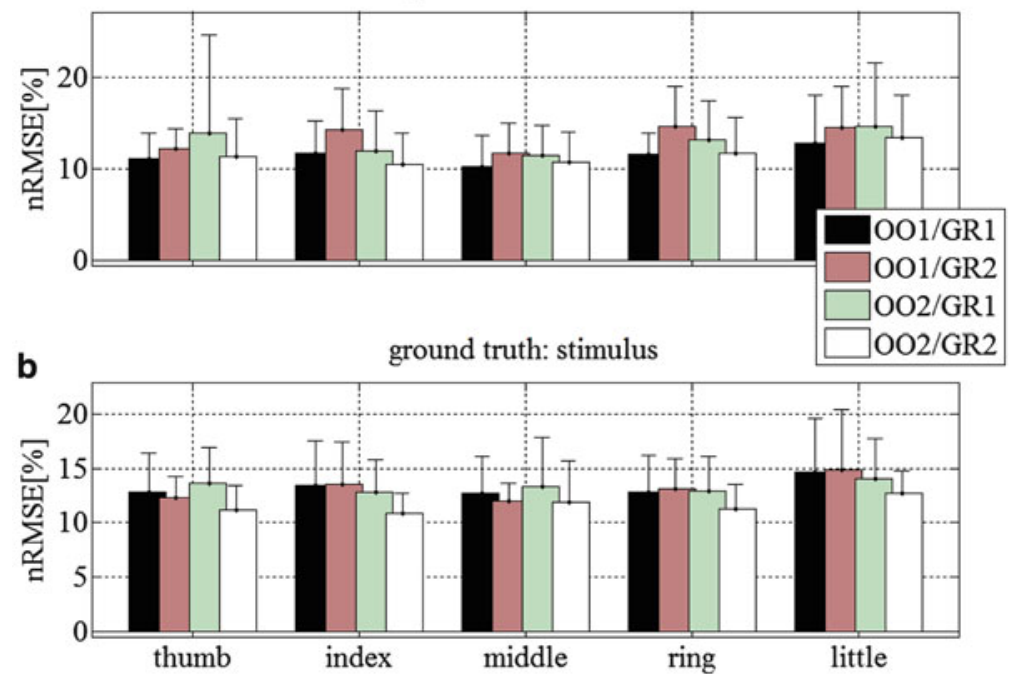

Fig. 2.10 Normalised root-mean-square error when training on an on-off dataset and testing on graded forces. The legend denotes the training/testing datasets, e.g., OO1/GR2 means that leastsquares regression was evaluated with data gathered during the first on-off phase and the prediction error was evaluated on data gathered during the second graded phase. (Top panel) With the force as ground truth; (bottom panel) with the stimulus as ground truth. Each bar and stem represents the mean nRMSE and one standard deviation obtained over 10 intact subjects

distinction between the training and the prediction phase, typical of more complex machine learning methods. Such a system is strictly bound in space and is therefore independent of the time spent while adapting to the subject.

Consider Eq. 2.2 again, and define for simplicity $\mathbf{w}=A \mathbf{b}$ where $A \triangleq\left(X^{T} X+\right.$ $\left.\lambda I_{d}\right)^{-1}$ and $\mathbf{b} \triangleq X^{T} \mathbf{y}$. As a new (sample,target) pair $\left(\mathbf{x}^{\prime}, y^{\prime}\right)$ is acquired, the updated regression vector $\mathbf{w}^{\prime}$ can be obtained by juxtaposing the new sample to $X$ and $\mathbf{y}$

$$
X^{\prime}=\left[\begin{array}{c}
X \\
\mathbf{x}^{\prime}
\end{array}\right] \quad \text { and } \quad \mathbf{y}^{\prime}=\left[\begin{array}{c}
\mathbf{y} \\
y^{\prime}
\end{array}\right]
$$

and then calculating $A^{\prime}$ and $\mathbf{b}^{\prime}$. The interesting part is that there is no need to compute the inverse of $\left(X^{T} X+\lambda I_{d}\right)$ at each such update, as it happened in Eq. 2.2, since $A$ can be directly updated using a rank-1 update, e.g., by using the ShermanMorrison formula [25]:

$$
A^{\prime}=A-\frac{A \mathbf{x}^{\prime} \mathbf{x}^{\prime T} A}{1+\mathbf{x}^{\prime T} A \mathbf{x}^{\prime}} \text { and } \mathbf{b}^{\prime}=\mathbf{b}+\mathbf{x}^{\prime} y^{\prime}
$$

As expected, adding a new sample will not increase the size of $A$, which always remains of size $d \times d$ - the system is strictly bound in space, irrespective of how 

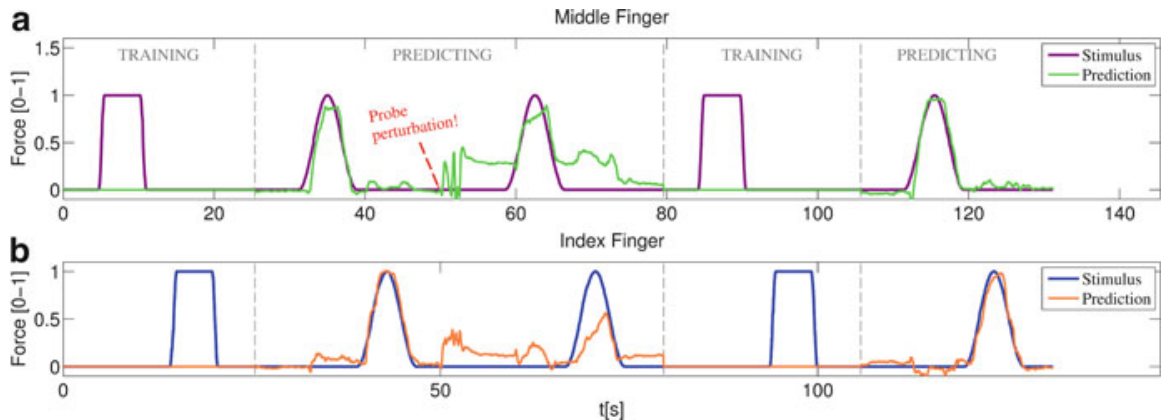

Fig. 2.11 Incremental training/testing in the experiment of [45]. After a perturbation in the position of the US probe reduces the quality of the prediction, new knowledge is acquired by the system. The prediction quickly recovers the accuracy obtained with the initial training. (a) Stimulus and prediction values for the middle finger; (b) stimulus and prediction values for the index finger

many updates are done. The time complexity of a rank-1 update is $\mathrm{O}\left(d^{2}\right)$, again independent of the total number of samples acquired so far, $n$. The net result is that the linear model can be updated each time new knowledge is available or is required, as neither the time- nor the space complexity grow as new samples are acquired. The system can switch back and forth from training to prediction and can then improve over time, as soon as the subject claims that the prediction is no longer accurate, or external events (e.g., a sudden movement of the ultrasound probe) happen.

Both a simulated online environment and a real implementation of the system were presented in [45]; Fig. 2.11 shows the temporal behaviour of the accuracy of the prediction, in the presence of an external disturbance and the subsequent acquisition of new knowledge to take that into account. The average timings reported therein for updating the linear model and predicting new values are, in turn, of 16.5 and $3.7 \mathrm{~ms}$.

\subsection{Discussion}

\subsubsection{Ultrasound Imaging as a Human Machine Interface}

The extensive experiments conducted in the works summed up in this chapter indicate that medical ultrasound imaging has the potentiality to become a fullyfledged HMI for the disabled, at least as far as hand amputees are concerned.

Sonomyography (Sect. 2.3) is being studied since 2006, and has been employed to control a one-degree-of-freedom wrist prosthesis; its prediction of the wrist angle during extension shows a high correlation coefficient with the ground truth; 
experiments with amputees are reported as promising. An interesting issue explored in this research is that of employing 1-dimensional (A-mode) ultrasonography, by means of single ultrasound transducers placed on the subject's forearm. These transducers are as large as standard sEMG electrodes and could potentially be embedded in an instrumented silicon liner or prosthetic socket, much as it happens with that kind of electrodes. Sonomyography represents then a very promising ongoing attempt at employing medical ultrasonography as an HMI, albeit so far restricted to large musculoskeletal structures. It may take some time to fully demonstrate the potential of sonomyography, given the fact that conventional medical US equipments are not designed for this purpose. For practical HMI applications using sonomyography, a number of challenges should be overcome, including probe miniaturization, probe attachment on skin, complexity of data collection system, signal processing for extracting meaningful data, multiple channel operation, and cost of system. Considering that US can provide not only signal but also crosssectional image of muscle, more information can be extracted for the purpose of HMI. For this point, the above reviewed studies of sonomography are just the beginning of the field, in both method development and application.

Furthermore, in Sect. 2.4, it is shown that a surprisingly simple system, based upon linear regression, can be used to predict the angles at the metacarpophalangeal hand joints, and the forces at the fingertips of a human intact subject. The precision is of the order of magnitude of $2 \%$ of the signal ranges when a dataglove or a force sensor are used; it reaches 10-15\% of the range when a visual stimulus is used. Furthermore, it is shown that a simple on-off task is enough to predict graded forces, and that no sensors are required to complete the calibration phase. This goes in the direction of providing a realistic scenario for the usage of such a device by amputees. Lastly, by exploiting the linearity of the relationship, an incremental system has been demonstrated, able to seamlessly run at $30 \mathrm{~Hz}$ and switch between training and prediction, paving the way to add new knowledge whenever required by the subject.

\subsubsection{Advantages, Disadvantages, Perspectives}

HMIs for the disabled are currently an extremely hot topic in both neuroscience, signal processing, machine learning and - of course - rehabilitation robotics. As is said in the introduction to this chapter, the only widely and practically used HMI for hand amputees is, currently, sEMG, which is however still far from representing the ideal solution to the problem of hand prosthesis feed-forward control. This is true to the point that many attempts at finding novel HMIs to replace or complement it have been carried out. Even if we limit ourselves to non-invasive HMIs, and to those HMIs which only interface to the peripheral nervous system (i.e., we do not consider brain-machine interfaces, computer vision, gaze tracking, etc.), we are faced with a plethora of different methods used to gather the intention of a patient from the forearm. These include, e.g., mechanomyography [27], magnetomyography [32] and 
pressure-based myography [48]. A thorough comparison among these techniques is still lacking, although their integration (fusion) is explicitly being called for:

Given the difficulty of robust control solely by using EMG, the use of other sensor modalities seems necessary for the control of complex devices. The rich multimodal input would not only allow for the improved control but could also lead to the development of intelligent controllers that are able to operate somewhat autonomously, thereby taking over some of the burden from the user. [...] inertial measurement units can measure the orientation and movement of the prosthesis and from this, the intention of the user and the phase of the reaching movement could be predicted, complementing the information obtained via a myoelectric interface. [...] The next steps in this direction should be the implementation of sensor-fusion approaches, in which inertial, myoelectric, and possibly other information sources (e.g., artificial vision) are integrated. [29]

The technique presented in this chapter is still too young to be fully compared with these alternative systems (although Zheng et al. already have provided some evidence of its competitiveness with respect to sEMG, see [22, 23, 26]). Nevertheless we think that medical ultrasound imaging will become one such source of information in the medium term. This claim is supported by its simplicity of use (from the point of view of computation) and by the precision it can achieve while predicting positions and forces associated with muscular activity. The main advantage of US imaging lies in its high spatial and temporal resolution, which enables the detection of tiny changes in the musculoskeletal structure. Actually, the prediction of finger positions is tantamount to the prediction of the small forces involved in free movement, which sEMG can probably hardly detect.

Of course, the development of US imaging as an HMI is in its early stages and a number of disadvantages must still be taken into account. Firstly, the fact that the B-mode ultrasonography is not yet embeddable in a prosthetic socket, although it has recently become portable (see the Background section). As far as A-mode ultrasound is concerned, such embedding of single ultrasonic transducers presents the non-trivial technological issue of the miniaturisation of the electronics required to form the ultrasonic beam. Secondly, although ultrasonic scanning has been declared harmless (see the Introduction), the medium-term effects of it on human tissue are still unknown. Continually injecting ultrasonic waves in the stump of an amputee for, e.g., 6-12 h might be detrimental and induce biological damage.

Therefore, there is probably still some time before US imaging will be embedded in a hand prosthesis. Meanwhile however, there is a further interesting possibility. The feasibility of US imaging as a means to predict finger positions opens up the scenario of relieving several forms of neuropathic pain. The approach is probably not limited to amputees, but also to a wide range of muscle/nerve injury patients; actually, in all cases in which the motor commands do not result any longer in coherent sensorial feedback pattern.

More in detail: with the term imaginary limb we mean here the intended configuration of the hand; i.e., what the patient wants the hand to do. This is in contrast to the so-called phantom limb, that is the residual sensorial image of the missing limb. It is well known that the two limbs very seldom coincide, due to the peripheral and central nervous system reorganisation that regularly happens after an 
amputation; the phantom limb is usually felt as cramped in an unusual or impossible position. This discrepancy in the sensori-motor feedback loop is thought to be at the basis of, e.g., phantom-limb pain; this is a current opinion in neuroscience and stems from Flor et al.'s [20] and and Ramachandran et al.'s [40] seminal works. Complex regional pain syndrome [33] is thought to be another manifestation of this problem.

Medical ultrasound imaging could then be used to visualise the imaginary limb and showing it to the patient in real time. One could think of this as a psychophysical treatment, akin and much superior to mirror therapy [9], in which a good visual illusion of the missing hand would alleviate the pain, when administered regularly over time.

Acknowledgements We gratefully acknowledge Dr. Yong-Ping Zheng of the Interdisciplinary Division of Biomedical Engineering, Hong Kong Polytechnic University, Hong Kong, China, for thoroughly reviewing the section about sonomyography.

We also acknowledge all colleagues involved in this topic, first and foremost David Sierra González, then Dr. Georg Passig, Emanuel Zarka, Thilo Wüsthoff, Santiago Pérez Chávez, Markus Nowak and Prof. Patrick van der Smagt.

\section{References}

1. Ali M, Magee D, Dasgupta U (2008) Signal processing overview of ultrasound systems for medical imaging. White paper, Texas Instruments, Inc.

2. Atkins DJ (1996) Epidemiologic overview of individuals with upper-limb loss and their reported research priorities. J Prosthet Orthot 8(1):2-11

3. Boser BE, Guyon IM, Vapnik VN (1992) A training algorithm for optimal margin classifiers. In: Haussler D (ed) Proceedings of the 5th annual ACM workshop on computational learning theory, Pittsburgh. ACM, pp 144-152

4. Bretscher O (2008) Linear algebra with applications, 4th edn. Pearson, London

5. Castellini C, Passig G (2011) Ultrasound image features of the wrist are linearly related to finger positions. In: Proceedings of IROS - international conference on intelligent robots and systems, San Francisco, pp 2108-2114

6. Castellini C, Sierra González D (2013) Ultrasound imaging as a human-machine interface in a realistic scenario. In: Proceedings of IROS - international conference on intelligent robots and systems, Tokyo

7. Castellini C, van der Smagt P (2009) Surface EMG in advanced hand prosthetics. Biol Cybern 100(1):35-47

8. Castellini C, Passig G, Zarka E (2012) Using ultrasound images of the forearm to predict finger positions. IEEE Trans Neural Syst Rehabil Eng 20(6):788-797

9. Chan BL, Witt R, Charrow AP, Magee A, Howard R, Pasquina PF, Heilman KM, Tsao JW (2007) Mirror therapy for phantom limb pain. N Engl J Med 357(21):2206-2207

10. Chen X, Zheng YP, Guo JY, Shi J (2010) Sonomyography (SMG) control for powered prosthetic hand: a study with normal subjects. Ultrasound Med Biol 36(7):1076-1088

11. Chen X, Chen S, Dan G (2011) Control of powered prosthetic hand using multidimensional ultrasound signals: a pilot study. In: Proceedings of the 6th international conference on universal access in human-computer interaction: applications and services - volume Part IV, UAHCI'11, Orlando. Springer, pp 322-327

12. Cobbold RSC (2007) Foundations of biomedical ultrasound. Biomedical engineering. Oxford University Press, Oxford/New York 
13. Cristianini N, Shawe-Taylor J (2000) An introduction to support vector machines (and other Kernel-based learning methods). Cambridge University Press, UK. http://www.amazon.com/ Kernel-Methods-Pattern-Analysis-Shawe-Taylor/dp/0521813972

14. De Luca CJ (1997) The use of surface electromyography in biomechanics. J Appl Biomech 13(2):135-163

15. Donald I, MacVicar J, Brown TG (1958) Investigation of abdominal masses by pulsed ultrasound. Lancet 271(7032):1188-1195

16. Douglas T, Solomonidis S, Sandham W, Spence W (2002) Ultrasound imaging in lower limb prosthetics. IEEE Trans Neural Syst Rehabil Eng 10(1):11-21

17. Dussik KT (1942) Über die möglichkeit, hochfrequente mechanische schwingungen als diagnostisches hilfsmittel zu verwerten (On the possibility of using ultrasound waves as a diagnostic aid). Zeitschrift für die gesamte Neurologie und Psychiatrie 174(1):153-168

18. Dussik KT, Fritsch DJ, Kyriazidou M, Sear RS (1958) Measurements of articular tissues with ultrasound. Am J Phys Med 37:160-165

19. Finley FR, Wirta RW (1967) Myocoder studies of multiple myopotential response. Arch Phys Med Rehabil 48(11):598-601

20. Flor H, Elbert T, Knecht S, Wienbruch C, Pantev C, Birbaumers N, Larbig W, Taub E (1995) Phantom-limb pain as a perceptual correlate of cortical reorganization following arm amputation. Nature 375(6531):482-484

21. Graff KF (1981) A history of ultrasonics. In: Mason WP, Thurston RN (eds) Physical acoustics, vol 15. Academic, New York, pp 1-99

22. Guo JY, Zheng YP, Huang QH, Chen X, He JF, Chan HLW (2009) Performances of onedimensional sonomyography and surface electromyography in tracking guided patterns of wrist extension. Ultrasound Med Biol 35(6):894-902

23. Guo JY, Zheng YP, Kenney LPJ, Bowen A, Howard D, Canderle JJ (2011) A comparative evalaution of sonomyography, electromyography, force, and wrist angle in a discrete tracking task. Ultrasound Med Biol 37(6):884-891

24. Guo JY, Zheng YP, Xie HB, Koo TK (2013) Towards the application of one-dimensional sonomyography for powered upper-limb prosthetic control using machine learning models. Prosthet Orthot Int 37(1):43-49

25. Hager W (1989) Updating the inverse of a matrix. SIAM Rev 31(2):221-239

26. Huang Q, Zheng Y, Chen X, He J, Shi J (2007) A system for the synchronized recording of sonomyography, electromyography and joint angle. Open Biomed Eng J 1:77-84

27. Islam MA, Sundaraj K, Ahmad RB, Ahamed NU (2013) Mechanomyogram for muscle function assessment: a review. PLoS ONE 8(3):e58902

28. Jensen JA (2002) Ultrasound imaging and its modeling. In: Fink M, Kuperman W, Montagner $\mathrm{J}$, Tourin A (eds) Imaging of complex media with acoustic and seismic waves. Topics in applied physics, vol 84. Springer, Berlin, pp 135-166

29. Jiang N, Dosen S, Muller K, Farina D (2012) Myoelectric control of artificial limbs - is there a need to change focus? IEEE Signal Process Mag 29(5):152-150

30. Jørgensen TM, Tycho A, Mogensen M, Bjerring P, Jemec GB (2008) Machine-learning classification of non-melanoma skin cancers from image features obtained by optical coherence tomography. Skin Res Technol 14(3):364-369

31. Kane D, Grassi W, Sturrock R, Balint PV (2004) A brief history of musculoskeletal ultrasound: "from bats and ships to babies and hips". Rheumatology 43(7):931-933

32. Mackert BM (2004) Magnetoneurography: theory and application to peripheral nerve disorders. Clin Neurophysiol 115(12):2667-2676

33. Maihöfner C, Baron R, DeCol R, Binder A, Birklein F, Deuschl G, Handwerker HO, Schattschneider J (2007) The motor system shows adaptive changes in complex regional pain syndrome. Brain 130(10):2671-2687

34. McDonald DG, Leopold GR (1972) Ultrasound B-scanning in the differentiation of baker's cyst and thrombophlebitis. Br J Radiol 45:729-732 
35. Merletti R, Botter A, Troiano A, Merlo E, Minetto M (2009) Technology and instrumentation for detection and conditioning of the surface electromyographic signal: state of the art. Clin Biomech 24:122-134

36. Micera S, Carpaneto J, Raspopovic S (2010) Control of hand prostheses using peripheral information. IEEE Rev Biomed Eng 3:48-68

37. Peerdeman B, Boere D, Witteveen H, in 't Veld RH, Hermens H, Stramigioli S, Rietman H, Veltink P, Misra S (2011) Myoelectric forearm prostheses: state of the art from a user-centered perspective. J Rehabil Res Dev 48(6):719-738

38. Ping H, Xue K, Murka P (1997) 3-D imaging of residual limbs using ultrasound. J Rehabil Res Dev 34(3):269-278

39. Rahmatullah B, Papageorghiou A, Noble J (2012) Image analysis using machine learning: anatomical landmarks detection in fetal ultrasound images. In: IEEE 36th annual computer software and applications conference (COMPSAC), 2012, Izmir, pp 354-355

40. Ramachandran VS, Rogers-Ramachandran D, Cobb S (1995) Touching the phantom limb. Nature 377(6549):489-490

41. Sayed AH (2008) Adaptive filters. Wiley/IEEE, Hoboken

42. Shi J, Chang Q, Zheng YP (2010) Feasibility of controlling prosthetic hand using sonomyography signal in real time: preliminary study. J Rehabil Res Dev 47(2):87-98

43. Shi J, Hu S, Liu Z, Guo J, Zhou Y, Zheng Y (2010) Recognition of finger flexion from ultrasound image with optical flow: a preliminary study. In: Proceedings of the international conference on biomedical engineering and computer science (EMBC), Wuhan, pp 1-4

44. Shi J, Guo J, Hu S, Zheng Y (2012) Recognition of finger flexion motion from ultrasound image: a feasibility study. Ultrasound Med Biol 38(10):1695-1704

45. Sierra González D, Castellini C (2013) A realistic implementation of ultrasound imaging as a human-machine interface for upper-limb amputees. Front Neurorobot 7:17

46. World Health Organisation (1998) Training in diagnostic ultrasound: essentials, principles and standards: report of a WHO study group. WHO technical report series, nr. 875, World Health Organisation

47. Xie H, Zheng Y, Guo J, Chen X, Shi J (2009) Estimation of wrist angle from sonomyography using support vector machine and artificial neural network models. Med Eng Phys 31(3): 384-391

48. Yungher DA, Wininger MT, Barr J, Craelius W, Threlkeld AJ (2011) Surface muscle pressure as a measure of active and passive behavior of muscles during gait. Med Eng Phys 33(4): 464-471

49. Zarka ER (2011) Prediction of finger movements using ultrasound images. Master thesis, DLR - German Aerospace Center, Germany and University of Applied Sciences Technikum Wien, Austria

50. Zhang J, Ma K, Er M, Chong V (2004) Tumor segmentation from magnetic resonance imaging by learning via one-class support vector machine. In: International workshop on advanced image technology (IWAIT '04), Singapore, pp 207-211

51. Zheng Y, Chan M, Shi J, Chen X, Huang Q (2006) Sonomyography: monitoring morphological changes of forearm muscles in actions with the feasibility for the control of powered prosthesis. Med Eng Phys 28:405-415

52. Zhou G, Zheng YP (2012) Human motion analysis with ultrasound and sonomyography. In: Proceedings of the international conference on biomedical engineering and computer science (EMBC), San Diego, pp 6479-6482 